\title{
Reoferesi nelle malattie del microcircolo
}

\author{
Rodolfo Puccini
}

Nefrologo, Metato (PI)

\begin{abstract}
RHEOPHERESIS AND PATHOLOGY OF THE MICROCIRCULATION
Abstract. The peripheral arterial disease (PAD) and the critical limb ischemia (CLI) are frequent and serious pathologies in dialysis, in both diabetic and non-diabetic patients, and represent serious clinical and social problems. Patients having serious trophic injuries often have to recur to amputation, despite the use of all the therapies that are currently available. In this article we attempt to underline the importance of the microcirculation and rheopheresis in affecting the prognosis of this pathology.
\end{abstract}

Key words: Peripheral arterial disease, Critical limb ischemia, Plasma viscosity, Rheopheresis, Microcirculation

Conflict of interest: None.

Financial support: None.

Ricevuto: 20 Gennaio 2013; Accettato: 5 Marzo 2013

\section{Microcircolo e Reoferesi}

L'arteriopatia periferica nei pazienti diabetici e non diabetici e uremici in trattamento dialitico o in trattamento conservativo è una patologia comune che colpisce una discreta percentuale della popolazione ed è un fenomeno in crescendo in considerazione dell'invecchiamento della popolazione.

L'incidenza della PAD e della CLI rende questa patologia una vera emergenza sociale: stime recenti danno questa patologia in 500-1000 individui per milione di abitanti. Circa il 15-20\% dei pazienti con arteriopatia periferica progredirà dalla claudicatio intermittens all'ischemia critica nel decorso della patologia. La presenza di lesioni trofiche aumenta il rischio di amputazione.

I pazienti diabetici presentano un rischio 4 volte più alto di sviluppare la CLI, i pazienti uremici in dialisi hanno un rischio dieci volte più alto e, per i pazienti uremici e diabetici, il rischio è ancora maggiore. Il numero delle amputazioni viene calcolato intorno a 200 per 1000 pazienti all'anno.

A un anno di distanza dalla diagnosi di PAD, 1/4 dei pazienti è vivo senza sviluppo di CLI, il $30 \%$ ha subito l'amputazione di 1 arto, il $20 \%$ vive e convive con la CLI e il $20 \%$ è deceduto. Le procedure di rivascolarizzazione sono associate a una riduzione della mortalità ma a un maggior rischio di amputazione, come se il beneficio di questi interventi fosse solo temporaneo. La combinazione di ostruzione aterosclerotica dei grossi vasi (macrocircolo) degli arti inferiori con la patologia del microcircolo porta a un ridotto flusso del circolo capillare e a un ridotto apporto di ossigeno e di sostanze nutritive ai muscoli e alla cute degli arti coinvolti dalla patologia ischemica, conducendo, alla fine, alla formazione di ulcere e alla perdita di tessuto.
L'ostruzione delle grosse arterie riduce il flusso sanguigno e la pressione di perfusione distalmente all'ostruzione.

Il danno aterosclerotico della parete vasale riduce la funzione endoteliale dei grossi vasi e del microcircolo.

Il flusso sanguigno nel letto capillare e, quindi, la liberazione di ossigeno e di sostanze nutritive ai muscoli e alla cute sono regolati dalla differenza di pressione tra le arteriole e le venule e sono influenzati dal diametro dei capillari e dalla viscosità ematica. Questo rapporto è descritto dalla formula di HagenPoiseuille: se il flusso sanguigno è ridotto a livello dell'ostruzione arteriosa e se la ridotta funzione endoteliale non permette la vasodilatazione dei capillari, la viscosità ematica rimane l'unico fattore che può ancora influenzare il flusso sanguigno capillare.

Cambiamenti nel diametro vasale influiscono per una variabile di 4 sul flusso plasmatico (per esempio, un aumento del diametro vasale di due volte determina un aumento di flusso di 16 volte). Fino a che è possibile avere una risposta vasodilatatoria del sistema microcircolatorio, le variazioni della viscosità hanno un'importanza relativa.

Quando siamo di fronte a un sistema circolatorio aterosclerotico e patologico che non risponde agli stimoli vasodilatatori, solo le variazioni della viscosità possono migliorare il flusso sanguigno; in particolare, la riduzione della viscosità del sangue intero andrà a migliorare il flusso nel macrocircolo, mentre la riduzione della viscosità plasmatica (determinata dai livelli plasmatici di colesterolo totale, alfa-2-macroglobulina, fibrinogeno e $\operatorname{IgM}$ ) andrà a migliorare il flusso nel microcircolo.

Questo rappresenta il razionale per l'impiego, a fini terapeutici, di quei presidi che, rimuovendo dal plasma una o più componenti proteiche ad alto peso molecolare, rappresentate dalle 
low density lipoprotein, dal fibrinogeno, dalle alfa-2-macroglobuline e dalle IgM, inducono una rapida riduzione della viscosità creando le premesse per il miglioramento del flusso del microcircolo.

Il flusso del microcircolo è, inoltre, condizionato dalle variazioni della volemia che si hanno inevitabilmente durante il trattamento dialitico e che portano a importanti contrazioni del volume plasmatico con il conseguente aumento della viscosità e con il conseguente ulteriore peggioramento del flusso nel microcircolo o in quello che rimane del microcircolo.

Oltre ai ben conosciuti fattori di rischio maggiori per questa patologia quali il fumo, il diabete mellito, l'età avanzata, l'ipertensione arteriosa, l'iperlipidemia e l'iperomocisteinemia e l'alterazione del metabolismo calcio-fosforo, sono stati identificati diversi altri fattori di rischio, in particolare quelli che danno lo stato infiammatorio del paziente in dialisi: elevati livelli di PCR e aumento del fibrinogeno, dell'apolipoproteina B, della lipoproteina $\mathrm{A}$ e della viscosità plasmatica.

La terapia della PAD è rivolta all'eliminazione dei fattori di rischio, all'esercizio físico per lo sviluppo di circoli collaterali, al trattamento farmacologico con statine, cilostazolo antiaggreganti e anticoagulanti, al ripristino del flusso sanguigno con manovre di angioplastica o di rivascolarizzazione chirurgica e all'utilizzo di prostaglandine. Le terapie di rivascolarizzazione del macrocircolo trovano i loro limiti nella plurifocalità delle lesioni e sono, inoltre, condizionate, nel loro successo, dallo stato del microcircolo, le cui condizioni spesso sono tali da non permettere la riperfusione tissutale nonostante la risoluzione della stenosi del macrocircolo.

La Figura 1 mostra un capillare di un paziente uremico confrontato con un capillare normale.

Il letto capillare risulta ridotto di oltre il 50\%, risulta ridotto il diametro capillare e il flusso appare granulare invece che continuo. Un microcircolo così ridotto ha perso la risposta vasodilatatoria endoteliale e, per quanto gli possa arrivare un flusso sanguigno aumentato dopo le manovre di rivascolarizzazione delle grosse arterie, non potrà assolvere alla sua funzione originale.

Come precedentemente esposto, in questa situazione morfologica, solo una riduzione della viscosità plasmatica potrà far migliorare il flusso in quello che resta del circolo capillare e permettere di nuovo il nutrimento dei tessuti muscolari e della cute.

Il successo di questa terapia, prima la rivascolarizzazione e poi il trattamento per migliorare il flusso nel microcircolo (prostaglandine, se è ancora presente una risposta vasodilatatoria, e aferetico, per ridurre la viscosità plasmatica), è, ovviamente, condizionato dalla residua condizione del letto capillare.

La Plasmaferesi è la procedura che permette la rimozione delle proteine plasmatiche con la conseguente riduzione della viscosità plasmatica. Tecniche di rimozione selettiva permettono l'aferesi di componenti plasmatiche determinate.

La Reoferesi è un sistema sicuro ed efficace di filtrazione differenziata del plasma che si esegue utilizzando come

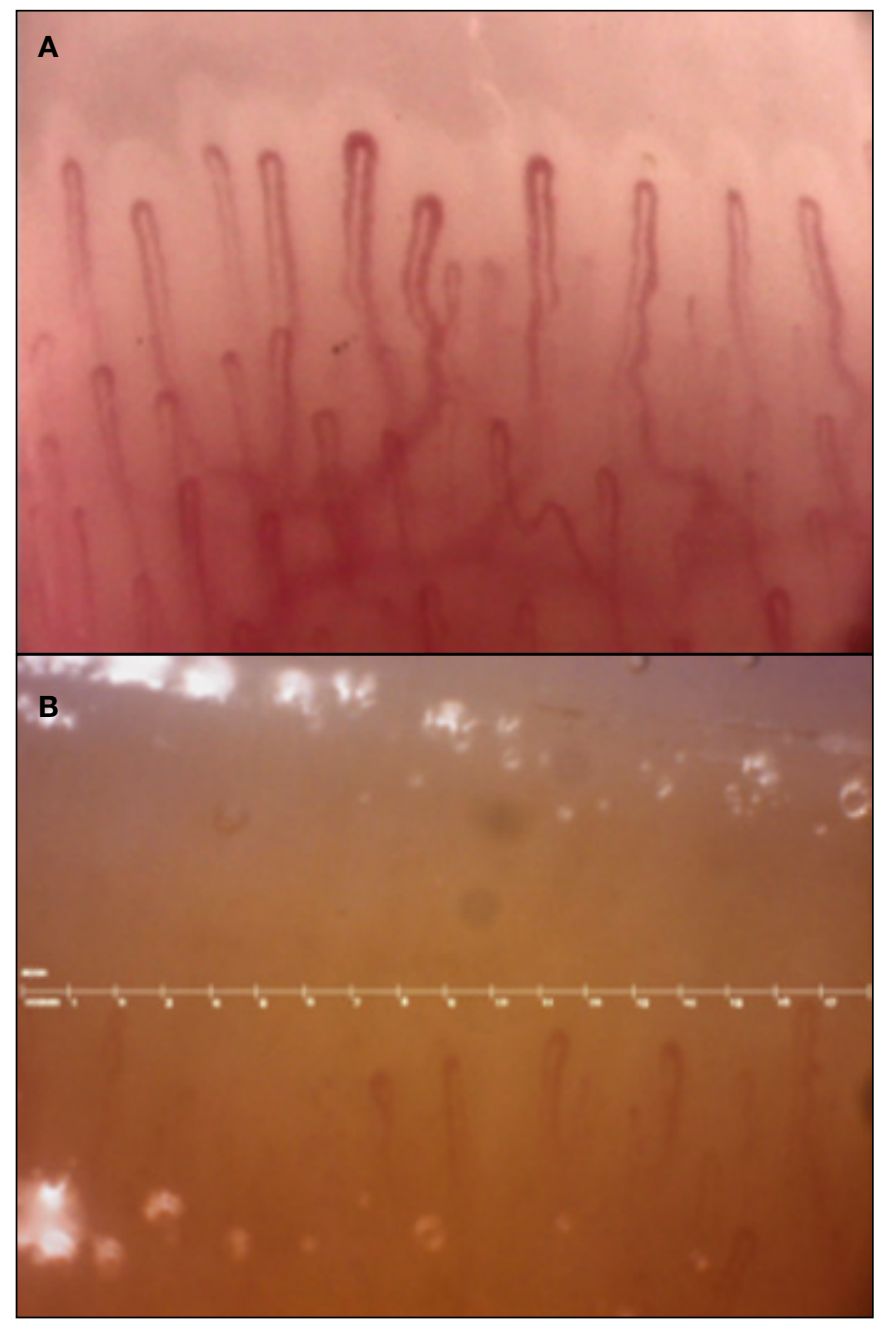

Fig. 1 - Il quadro capillaroscopico periungueale normale (A) è caratterizzato dalla presenza dei seguenti elementi:

- omogeneità morfologica, di diametro e di distribuzione dei capillari;

- aspetto a "U rovesciata" delle anse;

- flusso continuo;

- presenza di un capillare all'interno di ogni singola papilla dermica;

- rapporto tratto efferente:tratto afferente $<2: 1$;

- diametro 8-12 micron;

- assenza di atipie morfologiche;

- capillari presenti in $1 \mathrm{~mm}$ lineare 9-13.

Il quadro capillaroscopico patologico (B), riscontrato nei 7 pazienti in HD studiati, è caratterizzato da:

- 5 capillari per $\mathbf{m m}$ lineare;

- diametro capillare ridotto;

- flusso granulare.

secondo filtro uno speciale Plasmafiltro, chiamato Reofiltro, che elimina dal plasma uno spettro esattamente definito di molecole ad alto peso molecolare (fibrinogeno, alfa-2-macroglobulina, LDL-colesterolo, IgM).

I risultati sono una riduzione brusca, alla fine del trattamento, della viscosità plasmatica e un miglioramento dell'aggregazione e della flessibilità eritrocitarie. 


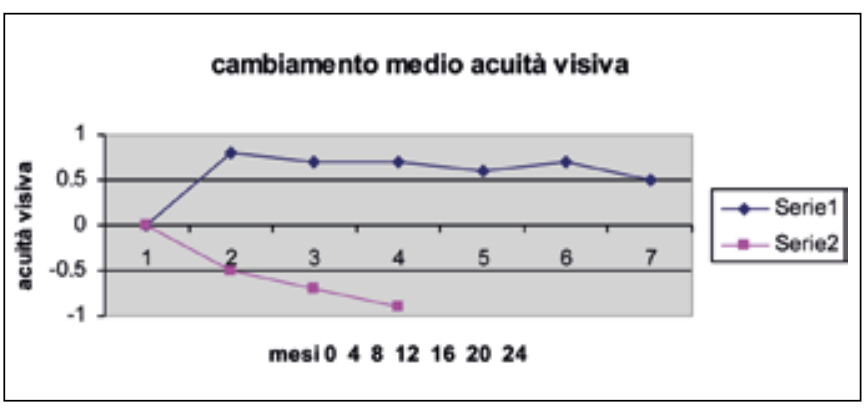

Fig. 2 - Serie 1: pazienti MDA trattati con Reoferesi (21 pazienti) Serie 2: gruppo controllo (10 pazienti con terapia tradizionale).

La riduzione della viscosità ematica e plasmatica e il miglioramento dell'aggregazione e della flessibilità eritrocitarie determinano un miglioramento del flusso ematico e il conseguente miglioramento del microcircolo, indipendentemente dalla malattia di base e dalle eventuali patologie collaterali.

A questo si associano sicuramente altri meccanismi tra cui la netta riduzione dei lipidi circolanti e un effetto benefico acuto e sub-acuto sulla funzione dell'endotelio microvascolare, come è stato riscontrato nei pazienti affetti da maculopatia degenerativa dell'anziano trattati con Reoferesi. La nostra esperienza nel trattamento di pazienti affetti da PAD e CLI (III e IV stadio, Fontaine) coincide con quanto descritto nel lavoro di Klinkel (1) che conclude: "Il presente studio pilota conferma l'impressione che una serie di Reoferesi possa avere un effetto positivo nei pazienti con CLI; i pazienti candidati al miglioramento sembrano quelli nello stadio Fontaine III e con $\mathrm{TcO} 2>30$. Al contrario, in quelli con lesioni trofiche già irreversibili e $\mathrm{TcO} 2<30$, la Reoferesi risulta inefficace" (2).

L'efficacia della Reoferesi nel trattamento delle patologia del microcircolo è stata estesamente valutata nella maculopatia degenerativa dell'anziano (MDA) (5). Le conclusioni dei molti studi pubblicati corrispondono a quelle a cui anche noi siamo giunti e indicano un'apparente reale efficacia non solo bloccando l'evoluzione della malattia ma inducendo anche un miglioramento dell' acuità visiva. Abbiamo trattato 21 pazienti, 14 femmine e 7 maschi, età media 72 anni. Tutti i pazienti sono arrivati alla nostra osservazione con la diagnosi di degenerazione maculare legata all'età, forma secca. Sia i pazienti trattati con Reoferesi che quelli del gruppo controllo seguivano anche la terapia medica convenzionale. La randomizzazione è stata interrotta dopo i primi 10 pazienti (in accordo con il comitato etico), per un'evidente e significativa differenza nell'evoluzione della patologia nei due gruppi. Nei pazienti trattati, abbiamo assistito a un miglioramento dell'acuità visiva, che si è mantenuto nei mesi e negli anni successivi (Fig. 2). Tre pazienti, dopo circa un anno e mezzo, hanno ripetuto il ciclo di Reoferesi. Nel gruppo controllo, al contrario, abbiamo assistito a un progressivo e lento peggioramento dell'acuità visiva.

Poco esiste in Letteratura relativamente alla patologia del microcircolo degli arti inferiori trattata con Reoferesi. Gli studi riportano risultati che è difficile attribuire alle manovre di rivascolarizzazione, alla terapia farmacologica e/o alla terapia aferetica, a ciascuno di questi presidi o all'insieme dei trattamenti e sono riferiti in particolare alla lipoaferesi in pazienti con gravi iperlipidemie.

Abbiamo avuto la possibilità di valutare l'effetto della Reoferesi sulla patologia del microcircolo degli arti inferiori in assenza di una patologia ostruttiva del macrocircolo, in una paziente uremica in dialisi presso il nostro centro, studiata 6 anni fa e 1 anno fa.

Paziente di 66 anni, in dialisi dal 1978 per ipertensione arteriosa; nel 2005 primo ciclo di Reoferesi per un quadro di Fontaine II senza patologia del macrocircolo. Al termine di 10 trattamenti di Reoferesi, netto miglioramento clinico e normalizzazione della $\mathrm{TcO} 2(<10$ prima del trattamento). Nel Febbraio del 2012 nuovo ciclo di 10 Reoferesi per la ricomparsa di una sintomatologia tipo Fontaine II. Il controllo eseguito circa due mesi dopo la fine del trattamento ha evidenziato a livello dell'alluce destro un netto miglioramento ossimetrico, situazione che è, invece, risultata invariata a sinistra. Capnometria bilaterale nella norma. Buon recupero bilaterale ossimetrico in ortostatismo. Netto miglioramento clinico. La capillaroscopia eseguita prima del trattamento e dopo è risultata invariata. Questo caso affetto solo da una patologia del microcircolo tende a dimostrare, a nostro avviso, l'efficacia della Reoferesi nel trattamento della patologia microcircolatoria. Assenza di variazioni morfologiche del letto capillare dopo il ciclo di aferesi; il miglioramento dei parametri sopradescritti alla fine del ciclo di Reoferesi, in assenza di variazioni morfologiche del letto capillare, può essere attribuibile, a nostro avviso, al miglioramento della perfusione capillare secondaria alla netta riduzione della viscosità plasmatica.

Per concludere, riteniamo che sia indispensabile uno studio sul potenziale effetto a breve e a lungo termine della Reoferesi nei pazienti con CLI. Auspico, pertanto, l'istituzione di un gruppo di studio multidisciplinare e multicentrico per valutare i parametri indispensabili per una stadiazione della lesione macro e microcircolatoria nella PAD e per coordinare uno studio clinico (come già condotto per la Maculopatia degenerativa dell'anziano), con un adeguato numero di pazienti e un adeguato follow-up che cerchi di stabilire la relazione tra Reoferesi e incidenza di amputazioni e mortalità e che cerchi anche di stabilire se la Reoferesi possa rappresentare un'opzione terapeutica nella pratica clinica per la CLI e/o per la sua prevenzione.

\section{Riassunto}

La peripheral arterial disease (PAD) e la critical limb ischemia (CLI) sono una frequente e grave patologia dei pazienti in dialisi, diabetici e non diabetici, e rappresentano un grave problema clinico e sociale. I pazienti che si pre- 
sentano con gravi lesioni trofiche arrivano frequentemente all'amputazione, nonostante l'utilizzo di tutti i presidi terapeutici oggi a nostra disposizione. In questo lavoro cerchiamo di sottolineare l'importanza del microcircolo e della Reoferesi nel condizionare la prognosi di questa patologia.

Parole chiave: Malattia arteriosa periferica, Ischemia critica degli arti inferiori, Viscosità plasmatica, Reoferesi, Microcircolo

Dichiarazione di conflitto di interessi: L'Autore dichiara di non avere conflitto di interessi.
Contributi economici degli autori: L'Autore dichiara di non aver ricevuto sponsorizzazioni economiche per la preparazione dell'articolo.

Indirizzo degli Autori:

Dr. Rodolfo Puccini

Via De Amicis 15

56010 Metato (PI)

puccinir@alice.it

\section{Bibliografia}

1. Klingel R, Erdtracht B, Gauss V, Piazolo A, Mausfeld-Lafdhiya P, Diehm C. Rheopheresis in patients with critical limb ischemia--results of an open label prospective pilot trial. Ther Apher Dial 2005; 9: 473-81.

2. Ferrannini M, Vischini G, Staffolani E, et al. Rheopheresis in vascular diseases. Int J Artif Organs 2007; 30: 923-9.

3. Ramunni A, Brescia P, Dambra P, et al. Effect of low-density lipoprotein apheresis on circulating endothelial progenitor cells in familial hypercholesterolemia. Blood Purif 2010; 29: 383-9.

4. Rossi M, Puccini R, Romagnoli MC, et al. Acute and subacute effect of rheopheresis on microvascular endothelial function in patients suffering from age-related macular degeneration. Ther Apher Dial 2009; 13 (6): 540-8.

5. Pulido J, Sanders D, Winters JL, Klinge R. Clinical outcomes and mechanism of action for rheopheresis treatment of age-related macular degeneration (AMD). J Clin Apher 2005; 20 (3): 185-94. 